\title{
Plasmodium falciparum histidine rich protein-2 diversity and the implications for PfHRP 2: based malaria rapid diagnostic tests in Ghana
}

\author{
Linda Eva Amoah*, Joana Abankwa and Akua Oppong
}

\begin{abstract}
Background: Malaria rapid diagnostic tests (RDTs) play a key role in malaria management and control. The PfHRP-2 based RDT is the most widely used RDT for malaria diagnosis in Ghana. Deletion of pfhrp2 in Plasmodium falciparum parasites affect the diagnostic accuracy of PfHRP-2 based RDT kits. Identifying the prevalence and distribution of $P$. falciparum parasites with deleted pfhrp2 is important for malaria control.
\end{abstract}

Aim: The purpose of this study was to identify and confirm the prevalence of pfhrp2 deletant $P$. falciparum parasites circulating within different regions of Ghana.

Methods: DNA was extracted from the membrane of spent CareStart ${ }^{\mathrm{TM}}$ PfHRP-2 RDT kits and dried filter paper blood blots using Chelex-100. Exon 2 of pfhrp2 and pfhrp3 genes were amplified by polymerase chain reaction (PCR), resolved by agarose gel electrophoresis and visualized under UV light.

Results: Microscopic analysis of blood smears from samples that were PfHRP-2 RDT positive revealed a parasite prevalence of 54/114 (47.4\%) and 2/26 (7.7 \%) in Accra and Cape Coast, respectively. PCR analysis increased parasite prevalence in the RDT positive samples to 94/114 (82.5\%) and 6/26 (23.1 \%) in Accra and Cape Coast respectively. The exon 2 of the pfhrp2 gene was deleted in 18/54 (33.3\%) of the microscopy confirmed and $36.2 \%$ (34/94) of the PCR confirmed RDT positive samples collected in Accra. No RDT sample, confirmed to contain parasites by either PCR or microscopy was negative by pfhrp2 exon 2 PCR in Cape Coast. A survey of an additional 558 DBS revealed that $22.4 \%$ (46/205) and $40 \%$ (44/110) of PCR positive samples in Accra and Cape Coast, respectively, lacked the exon 2 region of pfhrp2 and possibly the entire pfhrp2 gene.

Conclusions: A high number of P. falciparum parasites, which lack pfhrp 2 exon 2 gene have been identified in two communities in Ghana. Continuous nationwide monitoring of the prevalence of pfhrp2 deletant parasites would be essential to malaria control. The use of RDT kits that are effective at malaria diagnosis despite deletion of pfhrp2, such as the PfHRP-2/PfLDH combo RDT kit could enhance the diagnosis of clinical malaria in Ghana.

Keywords: Malaria diagnosis, Plasmodium falciparum, Rapid diagnostic test (RDT), Plasmodium falciparum histidine rich protein-2 (PfHRP-2), Plasmodium falciparum histidine rich protein-3 gene (pfhrp3)

\section{Background}

Malaria is one of the deadliest infectious diseases of humanity, which causes significant mortality and

*Correspondence: lamoah@noguchi.ug.edu.gh

Noguchi Memorial Institute for Medical Research, University of Ghana, Legon, Ghana morbidity in the tropics, particularly in Africa [1]. Malaria is a parasitic disease transmitted through the bite of an infectious female Anopheles mosquito. Early diagnosis is very important for disease management and the effective treatment of malaria. Before the advent of malaria rapid diagnostic tests (RDTs), diagnosis was based on microscopy of thick blood smears, which is 
still the gold standard for malaria diagnosis. However, in a number of rural and semi urban settings where lack of equipment, trained personnel and electricity prevents this essential diagnosis, health practitioners diagnose malaria based solely on clinical evaluation of symptoms [2]. RDTs offer a great potential for rapid immediate diagnosis of malaria infections, which has led to prompt and appropriate treatment of the disease, particularly in highly endemic rural settings [3].

Presently there is a very large demand for malaria RDT kits, as the World Health Organization (WHO) has recommended its use and majority of National Malaria Control Programmes have accepted it as the first step in the diagnosis of malaria. Due to the importance of the results of this initial screen, the WHO has established two programmes, the Foundation for Innovative New Diagnostics (FIND) malaria RDT quality assurance programme and WHO-FIND malaria RDT lot testing programme whose main mandate are to ensure accurate diagnosis of malaria [4]. Malaria RDT kits are designed to detect either Plasmodium falciparum specifically or discriminately detect both $P$. falciparum in addition to another human malaria parasite or indiscriminately detect all human malaria parasites $[4,5]$. The main antigens that malaria RDT kits detect are PfHRP2, parasite lactate dehydrogenase (pLDH), and parasite aldolase (pAldo). PfHRP-2 is a $P$. falciparum specific antigen with the advantage of being highly abundant and heat stable however, the PfHRP-2 antigen remains in circulation for up to 4 weeks after the malaria parasites have cleared $[6,7]$. Some monoclonal antibodies directed against PfHRP-2 have been found to cross react with PfHRP-3, a structural homologue of PfHRP-2 [8, 9]. Thus although PfHRP-2 based RDT kits have the highest sensitivities [4], they also have high false positive rates. By 2015, 171 different malaria RDT products had been tested by the WHO. Forty-five of these products detect only $P$. falciparum, ten detect $P$. falciparum as a part of a mixed infection with other human malaria parasites, one is Plasmodium vivax specific and 115 detect and distinguishing $P$. falciparum from either $P$. vivax mixed infections or mixed infections containing all the other human malaria parasites, $P$. vivax, $P$. ovale and $P$. malariae [10].

The accuracy of malaria RDT results can be affected by test antibody stability, product design and quality as well as the transport and storage conditions of the kits and sample parasite density [10]. Accurate diagnosis of malaria by PfHRP-2 RDT kits can be affected by the $p f h r p 2$ and or $p f h r p 3$ genotype of the parasite $[5,10,11]$, the amount of PfHRP-2 antigen produced by the parasite $[12,13]$ as well as the longevity of PfHRP-2 antigen after parasite clearance. One major obstacle in the diagnosis of malaria by RDT, without additional confirmation of parasitaemia is false positive test results, which leads to the unnecessary administration of anti-malarial drugs when no malaria parasites are actually present in the patient. False positive RDT test results are frequently obtained immediately following an anti-malarial drug regimen, when parasites are cleared or densities very low, but the antigen remains in circulation weeks later [14, 15].

In some facilities in Ghana, where microscopy is unavailable, malaria is treated based on RDT results. It is thus very important to monitor the accuracy of RDT results as well as identify factors that affect the diagnostic ability of malaria RDTs. So far the main studies conducted in Ghana have determined the sensitivity and specificity of different brands of malaria RDT kits, including the CareStart $^{\mathrm{TM}}$ and Paracheck RDT Kit [13, 16-18], in different cohorts of malaria This study systematically identifies and confirms the presence of $p f h r p 2$ deletant ( $p f h r p 2-$ ) parasites as well determines the prevalence of $P$. falciparum parasites with deletions in pfhrp2 and pfhrp3 (pfhrp3-) in two communities in Ghana.

\section{Methods}

\section{Ethics, consent and permissions}

This study was approved by the Institutional Review Board (IRB) of the Noguchi Memorial Institute for Medical Research, University of Ghana. Prior to enrollment, the study was explained to all participants after which written informed consent was obtained. Parental consent was obtained from parents and guardians of all children in addition to child assent obtained from children between 12 and 17 years.

\section{Study area and sample collection}

Abura Dunkwa, also known as Abura, is the district capital for Abura-Asebu-Kwamankese district and the Cape Coast Metropolis of the Central Region with a rural population of 31,768 for children under 14 years of age [19]. The Central Region is situated $165 \mathrm{~km}$ west of Accra (capital of Ghana). Malaria peak season coincides with the major rainy season between June through August. The community is a farming community.

Obom is in the Ga south municipality of the Greater Accra Region with a rural population of 22,368 for children under 14 years of age [20]. Malaria is perennial although it increases during the peak rainy season from June to August. The community is a fishing community. In 2014, malaria was estimated by microscopy to account for $35 \%$ of all out patient visits at the local Obom health centre.

The study utilized a total of 226 spent PfHRP-2 based RDT kits as well as 558 filter paper blood blots from consenting healthy children within the two study sites in 
2015. Thick and thin blood smears as well as DBS samples were obtained from healthy school children as part of a monthly malaria-screening programme from February through May. In April, RDT was performed according to manufacturers instructions in addition to the DBS and blood smears. The spent RDT cassettes were stored at room temperature for a maximum of 1 week, after which their membranes were processed for DNA. Approximately $50 \mu \mathrm{l}$ of finger-pricked blood was spotted on to filter paper to make the DBS and thick blood smears. DBS were kept in sealed plastic bags with a desiccant and stored at $-20{ }^{\circ} \mathrm{C}$ for no longer than 2 weeks after which they were processed for genomic DNA (gDNA). The slides containing the thick and thin blood smears were air dried and stored in slides boxes.

A sample was defined as negative by microscopy when two independent microscopists confirmed the absence of P. falciparum parasites on a Giemsa-stained thick blood smear. A sample was considered PCR positive when $P$. falciparum parasite genotyping using standard WHO genotyping procedures yielded a product. A positive RDT result was referred to as RDT positivity results, while a sample was considered positive for P. falciparum by RDT when the positive test strip was confirmed by microscopy or PCR. RDT positivity is used frequently as an indication of malaria in some facilities in Ghana where microscopy is unavailable.

\section{Microscopic estimation of malaria parasite}

Thick and thin blood smears as well as dried filter paper blood spots (DBS) were each made from a drop $(\sim 50 \mu \mathrm{l})$ of finger-prick blood. The blood smears were processed and then stained with $10 \%$ Giemsa for $15 \mathrm{~min}$. The stained slides were subsequently air-dried and viewed under 100X oil immersion microscope. Two independent microscopists read the slides and parasitaemia was determined as the \% of malaria parasite infected RBCs observed per 200 white blood cells (WBCs).

\section{Extraction of parasite DNA}

Genomic DNA was isolated from the membranes of the previously used PfHRP-2 RDT kits and dried filter paper blood spots (DBS) using either Tris-EDTA (TE) [21] or chelex [22]. Briefly, the RDT cassette was opened and portions between the filter paper through to the nitrocellulose membrane and some of the conjugated pad were cut and placed into a $1.5 \mathrm{ml}$ microcentrifuge tube containing $200 \mu \mathrm{l} \mathrm{TE}$; a separate scalpel was used for each RDT. Similarly, a $3 \mathrm{~mm}$ punch was used to punch two $3 \mathrm{~mm}^{2}$ disks from each of the dried blood spot (DBS). Each sample pieces was put into a $1.5 \mathrm{ml}$ microcentrifuge tube containing $200 \mu \mathrm{l} \mathrm{TE}$. The sample tubes were heated at $97{ }^{\circ} \mathrm{C}$ for $15 \mathrm{~min}$ on a dry heating block, centrifuged at $10,000 \mathrm{~g}$ for $30 \mathrm{~s}$ after which the supernatant transferred into a $500 \mu \mathrm{l}$ tube for storage at $-20^{\circ} \mathrm{C}$. For the chelex extraction, $150 \mu \mathrm{l}$ of $6 \%$ chelex in PBS was added to the tube with the punched DBS disks. The tubes were then incubated at $95{ }^{\circ} \mathrm{C}$ for $30 \mathrm{~min}$ with intermittent mixing by vortexing followed by a quick centrifugation step. The samples were centrifuged at $6000 \mathrm{~g}$ for $6 \mathrm{~min}$, after which $120 \mu \mathrm{l}$ of the supernatant was transferred into a $500 \mu \mathrm{l}$ tube for storage at $-20^{\circ} \mathrm{C}$.

\section{Plasmodium falciparum genotyping}

The WHO malaria parasite genotyping protocol [23] was followed with slight modifications. PCR reactions were carried out in $15 \mu \mathrm{l}$ volumes for both the primary and nested reactions. Briefly, the $200 \mathrm{nM} \mathrm{M} 2-0 \mathrm{~F}$ and M2-0R primers were used to amplify $4 \mu \mathrm{l}$ of gDNA using One Taq polymerase (NEB). The nested reaction was carried out using $1 \mu \mathrm{l}$ of the primary PCR product with $200 \mathrm{nM}$ each of the combination of S1Fw/N5rev for the 3D7 type alleles or S1Fw/M5rev for the FC27 type alleles. For GLURP, the G-F3 and G-F4 primer pair was used for the outer PCR reaction and the G-NF and G-F4 primer pair used for the nested inner reaction. All the PCR fragments and the digested products were viewed under UV after resolving on a $2 \%$ agarose gel containing $0.5 \mu \mathrm{g} / \mathrm{ml}$ ethidium bromide. Samples were classified as positive by PCR genotyping if the MSP2 and or GLURP PCR yielded a product following gel electrophoresis.

\section{PCR-based detection of pfhrp 2 and pfhrp 3 genes}

The PCR amplification was adapted from Baker et al. [23] with very minor modifications. Briefly, $2 \mu \mathrm{l}$ of gDNA was used as a template in a $20 \mu \mathrm{l}$ PCR reaction mixture that contained $200 \mathrm{mM}$ of each primer and 1X AmpliTaq Gold ${ }^{\circledR}$ Fast PCR Master Mix UP. The DNA was initially denatured at $96^{\circ} \mathrm{C}$ for 10 min followed by 41 cycles of denaturation at $95^{\circ} \mathrm{C}$ for $50 \mathrm{~s}$, annealing at $55^{\circ} \mathrm{C}$ for $50 \mathrm{~s}$ (pfhrp 2 gene) or $51{ }^{\circ} \mathrm{C}$ (pfhrp3 gene) and extension at $68{ }^{\circ} \mathrm{C}$ for $1 \mathrm{~min}$. The final extension was performed at $72{ }^{\circ} \mathrm{C}$ for 5 min then to $4{ }^{\circ} \mathrm{C}$. Genomic DNA from Dd2.

(pfhrp2-), HB3 (pfhrp3-) and 3D7 (wild type) were used as controls for the PCR amplifications. The primers used in the amplification of the exon 2 regions of $p f h r p 2$ and $p f h r p 3$ were $p f h r p 2-\mathrm{F} 1, p f h r p 2-\mathrm{F} 2$ and $p f h r p 2-\mathrm{R} 1$ for pfhrp2; pfhrp3-F1, pfhrp3-F2 and pfhrp3-R1 for $p f h r p 3$, as previously listed [23].

PCR amplification for all samples that gave a negative result for any primer set was repeated using twice the volume of gDNA as template. All PCR amplifications were either nested or semi nested. 


\section{Resolution of PCR amplicons by agarose gel electrophoresis}

PCR products were separated by electrophoresis on a $2.0 \%$ agarose gel stained with ethidium bromide in $1 \mathrm{X}$ TAE buffer. $10 \mu \mathrm{l}$ of PCR amplicons were loaded onto the gel, which was run for $1 \mathrm{~h}$ at $100 \mathrm{~V}$ then observed under UV light. The resolved fragment sizes were determined by comparison with $0.5 \mu \mathrm{g} / \mu \mathrm{l}$ Gene Ruler $100 \mathrm{bp}$ DNA ladder (Thermo Scientific) loaded on the same gel.

\section{Data analysis}

Crosstab descriptive analysis was performed using IBM SPSS Statistics (version 22). Microsoft Excel was used to draw the table and graphs.

\section{Results}

Plasmodium falciparum parasite carriage by microscopy Parasite carriage observed by microscopy in Obom (Accra) and Abura (Cape Coast) for February through May of 2015 was $41.2 \%(120 / 291)$ and $0.7 \%(2 / 267)$ respectively (Fig. 1a).
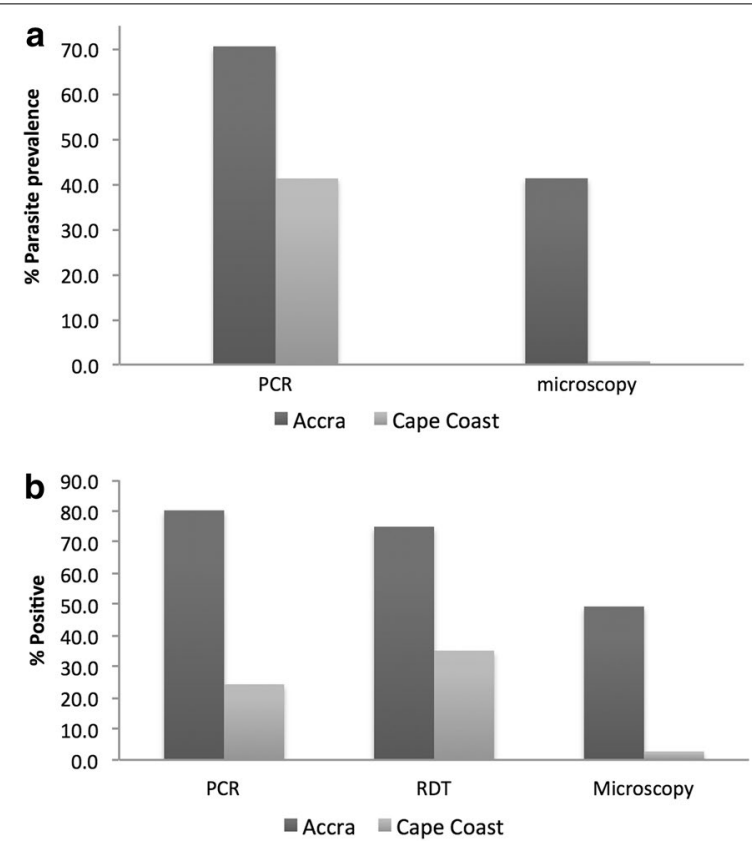

Fig. 1 Prevalence of Plasmodium falciparum in samples collected in 2015. a Giemsa-stained thick blood smears were read and parasite prevalence estimated by microscopy was compared with data obtained from the PCR genotyping of genomic DNA extracted from a DBS. Each Giemsa-stained blood smear analyzed had a corresponding DBS sample. $\mathbf{b}$ The frequencies of positivity (obtaining a positive test results) obtained by PCR, PfHRP-2 RDT and microscopy in the samples collected in April 2015 from Accra and Cape Coast

\section{Parasite genotyping by PCR}

A parasite carriage rate of $70.4 \%(205 / 291)$ and $41.2 \%$ $(110 / 267)$ was identified by PCR genotyping in Accra and Cape Coast respectively during the period of February through May 2015 (Fig. 1a).

In Accra, 46/205 (22.4\%) of the PCR positive samples contained $P$. falciparum parasites that lacked exon 2 of pfhrp2. Twenty percent (35/179) of the samples did not yield a product after PCR amplification and were classified as pfhrp3- (Table 1). Twenty-six of the PCR-positive samples collected in Accra were omitted from the pfhrp3 PCR analysis.

In Cape Coast, $40.4 \%(44 / 110)$ of the PCR positive samples contained $P$. falciparum parasites that lacked exon 2 of $p f h r p 2$. Forty-five percent (49/109) of the samples lacked exon 2 of pfhrp3. One sample was omitted in the pfhrp3 PCR analysis. Overall, $27.5 \%$ of the samples contained parasites that lacked exon 2 of both $p f h r p 2$ and pfhrp3 (Table 1).

\section{RDT positivity rate}

Two hundred and twenty six (226) spent PfHRP-2 RDT kits were obtained from school children in Accra and Cape Coast in April, 2015. Four of the test kits collected in Accra gave invalid results. An RDT positivity rate of $75 \%(114 / 152)$ was observed in Accra and $35.1 \%(26 / 74)$ in Cape Coast (Fig. 1b).

\section{Malaria estimation by PfHRP-2 RDT}

Microscopic examination of Giemsa-stained thick smears made from the same sample spotted onto the PfHRP-2 RDT kit identified P. falciparum in 54/152 (35.5\%) and 1/74 (1.4\%) of the samples from Accra and Cape Coast, respectively (Table 2). However PCR analysis of these RDT positive samples confirmed parasites in 94/114 (82.5\%) of the samples from Accra and 7/26 (26.9 \%) from Cape Coast (Table 2). False negative RDT results were obtained in $18 / 38(47.4 \%)$ of the negative branded RDT kits from Accra and 8/46 (17.4\%) of the negative branded RDT samples obtained from Cape Coast.

\section{Contributions of pfhrp2 and pfhrp3 to malaria diagnosis by PfHRP-2 RDT}

Genomic DNA from positive and negative branded PfHRP-2 RDT kits that were confirmed to contain $P$. falciparum by PCR genotyping were subjected to $p f h r p 2$ and pfhrp3 exon 2 PCR amplification.

In Accra, 34/94 (36 \%) of the PCR-confirmed RDT positive samples lacked pfhrp2 (Table 3), 30/34 (88.2\%) of these samples were positive for pfhrp3 and 4/34 (17.8\%) of the samples lacked both pfhrp 2 and pfhrp3 (Fig. 2). The prevalence of double $p f h r p 2-$ and $p f h r p 3-$ parasites 
Table 1 Prevalence of pfhrp2- and pfhrp3- parasites in samples collected from February to May 2015

\begin{tabular}{lccc}
\hline & pfhrp2-/pfhrp3- & pfhrp2-/pfhrp3+ & pfhrp2+/pfhrp3- \\
\hline Accra (179) & $3.9 \%(7)$ & $14 \%(25)$ & $16.2 \%(29)$ \\
Cape Coast (109) & $27.5 \%(30)$ & $12.8 \%(14)$ & $17 \%(19)$ \\
\hline
\end{tabular}

pfhrp2- no product obtained after pfhrp2 exon 2 PCR, pfhrp3 - no product obtained after pfhrp3 exon 2 PCR, pfhrp $2+$ a product was obtained after pfhrp2 exon 2 PCR, pfhrp3+ a product was obtained after pfhrp3 exon 2 PCR. Frequency of occurrence is stated in parenthesis alongside prevalence expressed as a percent of the total population of 179 in Accra and 109 in Cape Coast

Table 2 Comparison of microscopy, PCR genotyping and PfHRP-2 RDT results from the samples collected in April 2015

\begin{tabular}{lccc}
\hline & PCR- & PCR+ & $\begin{array}{c}\text { Micros- } \\
\text { copy+ }\end{array}$ \\
\hline Accra (RDT-) & $31.6 \%(12 / 38)$ & $68.4 \%(26 / 38)$ & $47.4 \%(18 / 38)$ \\
& $75 \%(36 / 48)$ & $25 \%(12 / 48)$ & $97.9 \%(47 / 48)$ \\
Cape Coast (RDT-) & $17.5 \%(20 / 114)$ & $82.5 \%(94 / 114)$ & $52.6 \%(60 / 114)$ \\
Accra (RDT+) & $73.1 \%(19 / 26)$ & $26.9 \%(1 / 48)$ & $(54 / 114)$ \\
Cape Coast (RDT+) & $3.8 \%(1 / 26)$ & $96.2 \%(25 / 26)$ \\
\hline
\end{tabular}

$P C R-P$. falciparum negative by $P C R$ genotyping, $P C R+P$. falciparum positive by $P C R$ genotyping, microscopy $-P$. falciparum negative by microscopy, microscopy + P. falciparum positive by microscopy, RDT+ sample produced a positive PfHRP-2 RDT test strip, RDT - sample produced a negative PfHRP-2 RDT test strip. A total of $114 / 152$ and $38 / 152$ positive and negative branded RDT kits respectively were collected from Accra and 26/74 positive and 48/74 negative branded RDT kits respectively were collected from Cape Coast

Table 3 Prevalence of pfhrp2 - in false negative PfHRP-2 RDT test determined by PCR

\begin{tabular}{lcl}
\hline & PfHRP-2 RDT- & PfHRP-2 RDT+ \\
\hline pfhrp2 PCR - (Accra) & $23 \%(6 / 26)$ & $36 \%(34 / 94)$ \\
pfhrp2 PCR+ (Accra) & $77 \%(20 / 26)$ & $64 \%(60 / 94)$ \\
pfhrp2 PCR - (Cape Coast) & $0 \%(0 / 12)$ & $14 \%(1 / 7)$ \\
pfhrp2 PCR+ (Cape Coast) & $100 \%(12 / 12)$ & $86 \%(6 / 7)$ \\
\hline
\end{tabular}

Samples that were confirmed as P. falciparum positive by PCR genotyping were grouped according to their PfHRP-2 RDT result, PfHRP-2 RDT positive (PfHRP-2 RDT+) or PfHRP-2 RDT negative (PfHRP-2 RDT-). pfhrp2 exon 2 PCR amplification was performed on each sample to estimate effect of the presence of absence of pfhrp2 on malaria diagnosis by PfHRP-2 RDT kits

increased from $4.3 \%(4 / 94)$ to $7.4 \%(4 / 54)$ in microscopy-confirmed RDT positive samples. The prevalence of pfhrp2-/pfhrp3+ samples however decreased from $31.9 \%(30 / 94)$ to $25.9 \%(14 / 54)$ (Fig. 2). No pfhrp2- parasite was identified in any of the RDT positive sample collected in Cape Coast.

\section{Prevalence of pfhrp2- and pfhrp3- parasites}

In an additional survey of gDNA extracted from DBS, the prevalence of $p f h r p 2-$ parasites that had intact pfhrp3 (pfhrp2-/pfhrp3+) was to be $14 \%(25 / 205)$ in Accra and $13 \%(14 / 110)$ in Cape Coast and the prevalence of parasites with intact $p f h r p 2$ that were $p f h r p 3-$ (pfhrp2+/pfhrp3-) was $16 \%(29 / 201)$ in Accra and $17 \%$ (19/109) in Cape Coast (Fig. 3).

\section{Discussion}

The recent recommendation for accurate classification of pfhrp2- parasites calls for an initial microscopic evaluation of the parasites, followed by Plasmodium speciesspecific PCR analysis, after which confirmation is carried out by $p f h r p 2$ specific gene amplification to determine the absence of the gene or antigen analysis using a second quality PfHRP-2 based RDT or PfHRP-2 based ELISA [24]. Many studies have reported the presence of pfhrp2- parasites in a number of South American countries [25-28], one study analysed 68 isolates and did not find any deletions in the pfhrp 2 gene nor its flanking sequences but rather found $50 \%$ of the isolates to have deletions in the pfhrp3 gene and its flanking genes [27]. Such variation between nearby countries raises the need for all malaria endemic countries to engage in nationwide pfhrp2 surveillance.

In Ghana, RDTs are used for malaria diagnosis throughout the year, during both peak and off peak seasons. A common practice in a number of health facilities is to rule out malaria in patients that test negative with an RDT kit, without further confirmation. This makes accurate malaria diagnosis using RDT kits very essential for malaria control.

The few previous studies on the PfHRP-2 based RDT kits in Ghana have focused on determining the sensitivity and specificity of Pf-HRP2 RDT kits [17, 29]. This study provides some preliminary evidence for the existence of pfhrp2- parasites as well as determines how mutant parasites with deletions in one or both $p f h r p 2$ and $p f h r p 3$ 

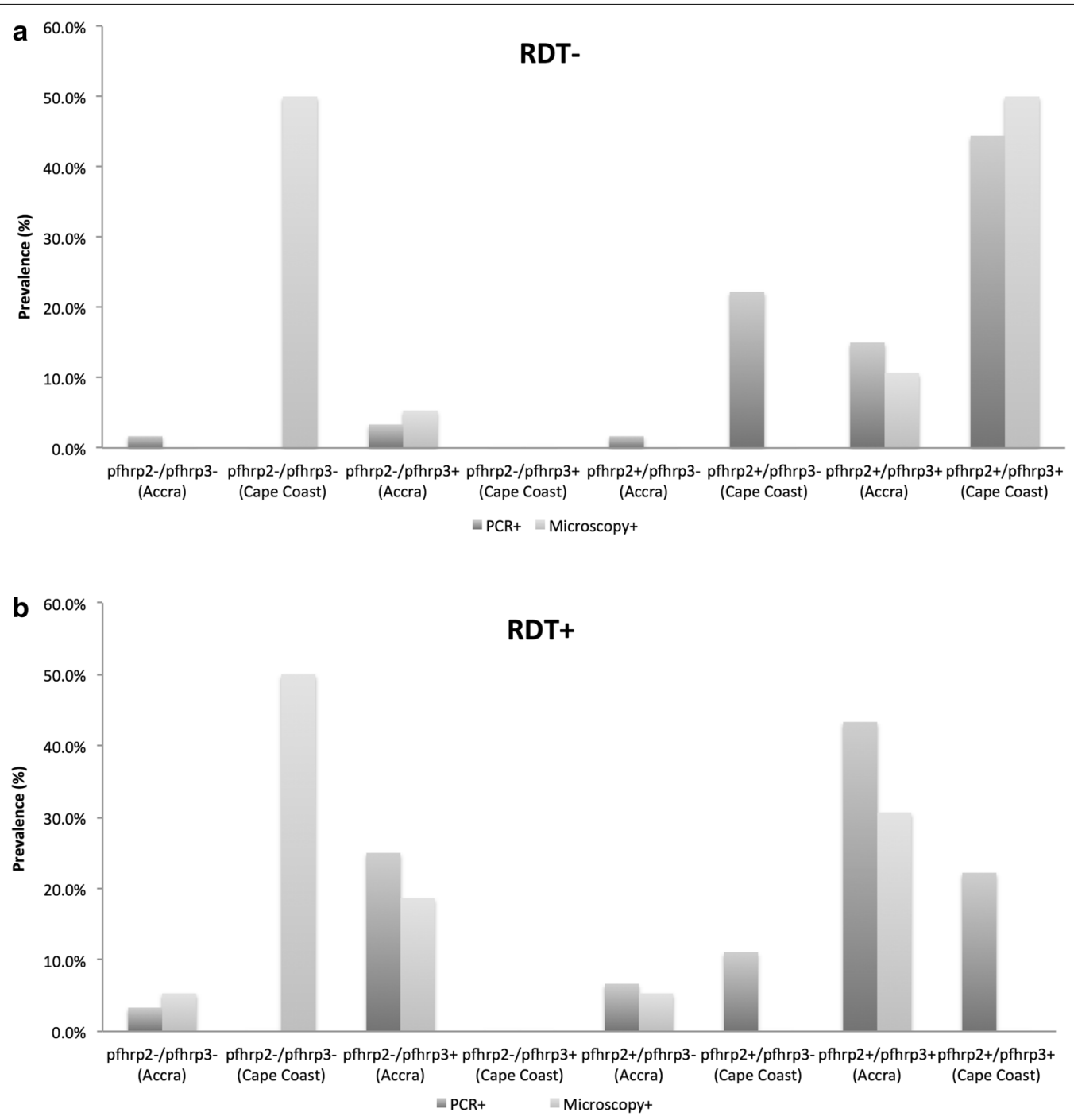

Fig. 2 Contributions of pfhrp2 and pfhrp3 to PfHRP-2 RDT read out. Genomic DNA obtained from either the membrane of the PfHRP-2 RDT kit or the corresponding DBS sample was subjected to pfhrp2 and pfhrp3 exon 2 PCR amplification. The presence or absence of pfhrp2 and or pfhrp3 in negative (a) and positive (b) banded PfHRP-2 RDT kits collected from Accra and Cape Coast was identified. Each RDT kit was confirmed as P. falciparum positive by PCR and microscopy

influence the accuracy of malaria diagnosis by PfHRP-2 RDT in Ghana.

Parasite prevalence estimated by PCR genotyping of $70 \%$ for February to May was almost twice what was estimated by microscopy of corresponding thick blood smears in Accra. In Cape Coast, parasite prevalence estimated by microscopy of $0.7 \%$ was only a small fraction of that estimated by PCR (41.2 \%) (Fig. 1a). This suggests that more sensitive diagnostic tools are needed to accurately diagnose malaria in settings with a high prevalence of sub microscopic parasites.

To estimate the true prevalence of pfhrp2parasites, which includes double pfhrp2- and pfhrp3- (pfhrp2-/pfhrp3-) parasites; each gDNA sample was analyzed by PCR genotyping prior to $p f h r p 2$ and pfhrp3 exon 2 PCR.

False positive PfHRP-2 RDT results are not uncommon in malaria endemic settings as the PfHRP2 antigen persists for weeks after parasite clearance; however, in Accra where $P$. falciparum parasite prevalence was high, the PCR estimate of parasite prevalence was comparable to the RDT positive rate (Fig. 1b). Despite the similar diagnostic read out between PCR genotyping and RDT, PCR confirmed the presence of $P$. falciparum in 94/114 of the RDT positive samples, suggesting a false positive rate of $17.5 \%(20 / 114)$. Microscopic evaluation of the 


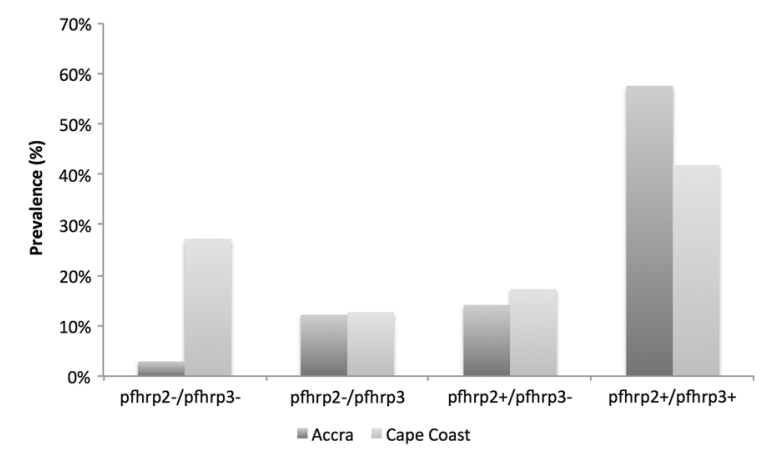

Fig. 3 Prevalence of $P$. falciparum parasites lacking exon 2 of pfhrp2 and or pfhrp3. Samples that were confirmed positive for $P$. falciparum by PCR genotyping were further analyzed for the presence of $p$ fhrp2 and pfhrp3 by PCR amplification of exon 2. Samples were grouped according to the presence or absence of either or both pfhrp 2 and pfhrp3

RDT samples increased the false positive rate to $60 / 114$ (52.6\%) in Accra. PCR genotyping identified 26/38 negative branded RDT kits to be positive for $P$. falciparum, out of these 26 samples, 12 were positive by microscopy.

In Cape Coast, the RDT positive rate was higher than parasite estimation by both microscopy and PCR, confirming PfHRP2 antigen persistence. There were only two positive microscopy slides over the entire 4 months (Table 2). The low prevalence and density of P. falciparum parasites causes the persistence of PfHRP2 to become more evident.

False negative RDT results are obtained when parasite carriage is confirmed by either microscopy or PCR, however the RDT kit produces a negative test results. This can have severe consequences in malaria endemic settings where negative RDT kit results are not confirmed by any other diagnostic tests such as microscopy. The prevalence of false negative RDT results increased from 18/38 when the samples collected in Accra were confirmed by microscopy to $26 / 38$ when confirmed by PCR (Table 2). This increase was due to PCR confirming more samples as parasite positive than microscopy. Twenty-three percent $(6 / 26)$ of the false negative samples carried deletions in the pfhrp 2 gene (Table 3 ), which suggests other factors including low parasite density contributed more to the negative RDT diagnosis than deletions in $p f h r p 2$.

In Cape Coast, PCR genotyping confirmed the presence of 12 false negative RDT tests. All 12 samples were positive for pfhrp 2 by exon 2 PCR, confirming our observation that factors other than $p f h r p 2$ deletion, including the high prevalence of submicroscopic parasites accounted for the false negative RDT results (Table 3).

Persistence of PfHRP-2 antigen from a recent past infection could explain the false positive RDT test, however possible compensation of $p f h r p 3$ for the lack of pfhrp 2 could also contribute to the positive RDT results obtained in samples that lacked pfhrp2. Eighty-eight percent of the samples from Accra that were pfhrp2- but were positive by PfHRP2 RDT and PCR genotyping were pfhrp3+ (Fig. 2). Although the sensitivity for the CareStart $^{\mathrm{TM}}$ PfHRP-2 RDT has proven to be very high in relation to microscopy in the recent WHO screen $[4,10]$ and between 100 and $96 \%$ in Ghana [16, 17]. During the offpeak malaria season, the prevalence of false negative tests was as high as $68.4 \%$ by PCR and $52.6 \%$ by microscopy. The specificity of the CareStart ${ }^{\mathrm{TM}}$ PfHRP-2 RDT has previously been found to be between 70 and $73 \%$ in Ghana $[16,17]$, however the false positive RDT results obtained in Accra were $17.5 \%$ by PCR and $52.6 \%$ by microscopy.

Double pfhrp2-/pfhrp3- parasites have been found to be as high as $25.7 \%$ in some countries within the Amazon basin $[25,28]$. The prevalence of parasites with pfhrp2-/pfhrp3- was $28 \%$ in samples obtained from Cape Coast over February through May (Fig. 3), however the subset of these samples that were analysed in April did not contain any double pfhrp2-/pfhrp3- parasite. The prevalence of double $p f h r p 2-/ p f h r p 3-$ parasites obtained in Accra over the months of February to May was $4.3 \%$, which was similar to $3 \%$ that obtained in the samples collected in April.

\section{Limitations}

This study was carried out in the off-peak malaria season, where $P$. falciparum prevalence and density is relatively low in most parts of Ghana. Although this is a major limitation, PfHRP-2 RDT kits are used for malaria diagnosis over this period, making this study highly important.

\section{Conclusion}

Plasmodium falciparum parasites that lack pfhrp 2 alone or in addition to pfhrp3 have been identified in two regions of Ghana based on the assumption that deletions in exon 2 of these genes represents deletions of the entire gene. Malaria RDT testing is highly suitable for diagnosis in communities where parasite densities are high but become less accurate when parasite densities are low and also where pfhrp 2 deletant parasites are prevalent. More accurate diagnosis of malaria would be obtained in countries such as Ghana where $p f h r p 2$ deletant parasites exits when a more sensitive RDT kit such as the pLDH/PfHRP-2 combo RDT kit that is able to detect $p f h r p 2$ deletant parasites is used. More studies are needed to evaluate RDT use in the peak malaria season as well as identify the possible influence $p f h r p 2$ and $p f h r p 3$ sequence diversity has on the diagnostic read out of PfHRP-2 based RDT kits across Ghana. 


\section{Abbreviations}

RDT: rapid diagnostic test; PfHRP-2: Plasmodium falciparum histidine rich protein-2; pfhrp2: Plasmodium falciparum histidine rich protein-2 gene; pfhrp3: Plasmodium falciparum histidine rich protein-3 gene; PCR: polymerase chain reaction; ELISA: enzyme linked immunosorbent assay; pfhrp2-: parasite with pfhrp2 deleted; pfhrp3-: P. falciparum with pfhrp3 deleted.

\section{Authors' contributions}

LEA, the PI for this study conceived and designed the study. JA and AO collected the samples and performed the experiments. LEA and JA wrote the manuscript. All authors read and approved the final manuscript.

\section{Acknowledgements}

We would like to thank all the volunteers who gave us consent to work on their samples as well as Access BIO for donating the PfHRP-2 RDT kits used in the study. We are also grateful to Drs. Nancy Duah and Kwadwo Asamoah Kusi of the Noguchi Memorial Institute for Medical Research (NMIMR) for providing various forms of assistance.

\section{Competing interests}

The authors declare that they have no competing interests.

\section{Received: 5 December 2015 Accepted: 10 February 2016} Published online: 18 February 2016

\section{References}

1. Skeet J. Malaria: its causes, treatment and methods of prevention. Nurs Times. 2005;101:43-5.

2. Bell D, Wongsrichanalai C, Barnwell JW. Ensuring quality and access for malaria diagnosis: how can it be achieved? Nat Rev Microbiol. 2006;4:682-95.

3. Makler MT, Palmer CJ, Ager AL. A review of practical techniques for the diagnosis of malaria. Ann Trop Med Parasitol. 1998;92:419-33.

4. WHO. WHO-FIND malaria RDT evaluation programme: product testing round 5. Geneva: World Health Organization; 2015.

5. WHO. Good practices for seleting and procuring rapid diagnostic tests for malaria. Geneva: World Health Organization; 2011.

6. lqbal J, Siddique A, Jameel M, Hira PR. Persistent histidine-rich protein 2, parasite lactate dehydrogenase, and panmalarial antigen reactivity after clearance of Plasmodium falciparum monoinfection. J Clin Microbiol. 2004;42:4237-41.

7. Kumar N, Singh JP, Pande V, Mishra N, Srivastava B, Kapoor R, et al. Genetic variation in histidine rich proteins among Indian Plasmodium falciparum population: possible cause of variable sensitivity of malaria rapid diagnostic tests. Malar J. 2012;11:298.

8. Lee N, Baker J, Andrews KT, Gatton ML, Bell D, Cheng Q, et al. Effect of sequence variation in Plasmodium falciparum histidine- rich protein 2 on binding of specific monoclonal antibodies: implications for rapid diagnostic tests for malaria. J Clin Microbiol. 2006;44:2773-8.

9. Lee N, Gatton ML, Pelecanos A, Bubb M, Gonzalez I, Bell D, et al. Identification of optimal epitopes for Plasmodium falciparum rapid diagnostic tests that target histidine-rich proteins 2 and 3. J Clin Microbiol. 2012;50:1397-405.

10. WHO. Malaria rapid diagnostic test performance: summary results of WHO product testing of malaria RDTs: rounds 1-6. (2008-2015).

11. Wurtz N, Fall B, Bui K, Pascual A, Fall M, Camara C, et al. Pfhrp2 and pfhrp3 polymorphisms in Plasmodium falciparum isolates from Dakar, Senegal: impact on rapid malaria diagnostic tests. Malar J. 2013;12:34.

12. Ho MF, Baker J, Lee N, Luchavez J, Ariey F, Nhem S, et al. Circulating antibodies against Plasmodium falciparum histidine-rich proteins 2 interfere with antigen detection by rapid diagnostic tests. Malar J. 2014;13:480.
13. Baiden F, Malm K, Bart-Plange C, Hodgson A, Chandramohan D, Webster $J$, et al. Shifting from presumptive to test-based management of malaria-technical basis and implications for malaria control in Ghana. Ghana Med J. 2014:48:112-22.

14. Gerstl S, Dunkley S, Mukhtar A, De Smet M, Baker S, Maikere J. Assessment of two malaria rapid diagnostic tests in children under five years of age, with follow-up of false-positive pLDH test results, in a hyperendemic falciparum malaria area, Sierra Leone. Malar J. 2010;9:28.

15. Adu-Gyasi D, Adams M, Amoako S, Mahama E, Nsoh M, Amenga-Etego S, et al. Estimating malaria parasite density: assumed white blood cell count of 10,000/mul of blood is appropriate measure in Central Ghana. Malar J. 2012;11:238.

16. Ansah E, Narh-Bana S, Affran-Bonful H, Bart-Plange C, Cundill B, Gyapong $M$, et al. The impact of providing rapid diagnostic malaria tests on fever management in the private retail sector in Ghana: a cluster randomized trial. BMJ. 2015;350:h1019.

17. Baiden F, Webster J, Tivura M, Delimini R, Berko Y, Amenga-Etego S, et al. Accuracy of rapid tests for malaria and treatment outcomes for malaria and non-malaria cases among under-five children in rural Ghana. PLoS One. 2012;7:e34073.

18. Achaempong DO. The efficacy of rapid diagnostic test (RDT) in diagnosing Plasmodium falciparum malaria in scome selected health facilities in the Cape Coast Metropolis of Ghana. Adv Appl Sci Res. 2011;2:348-56.

19. Government of Ghana. Multiple Indicator Cluster Survey 2011. Ghana Health Service, Accra, Ghana, UNICEF, UNFPA, Japan Official Development Assistance, USAID and ICF International, Calverton, Maryland, USA Ghana, 2012.

20. Ghana Statistical Service. Population by region, district, age groups and sex. 2012

21. Bereczky S, Martensson A, Gil JP, Farnert A. Rapid DNA extraction from archive blood spots on filter paper for genotyping of Plasmodium falciparum. Am J Trop Med Hyg. 2005;72:249-51.

22. Baidjoe A, Stone W, Ploemen I, Shagari S, Grignard L, Osoti V, et al. Combined DNA extraction and antibody elution from filter papers for the assessment of malaria transmission intensity in epidemiological studies. Malar J. 2013;12:272.

23. Baker J, McCarthy J, Gatton M, Kyle DE, Belizario V, Luchavez J, et al. Genetic diversity of Plasmodium falciparum histidine-rich protein 2 (PfHRP2) and its effect on the performance of PfHRP2-based rapid diagnostic tests. J Infect Dis. 2005;192:870-7.

24. Cheng Q, Gatton ML, Barnwell J, Chiodini P, McCarthy J, Bell D, et al. Plasmodium falciparum parasites lacking histidine-rich protein 2 and 3: a review and recommendations for accurate reporting. Malar J. 2014;13:283.

25. Gamboa D, Ho MF, Bendezu J, Torres K, Chiodini PL, Barnwell JW, et al. A large proportion of $P$. falciparum isolates in the Amazon region of Peru lack pfhrp2 and pfhrp3: implications for malaria rapid diagnostic tests. PLoS One. 2010;5:e8091.

26. Akinyi S, Hayden T, Gamboa D, Torres K, Bendezu J, Abdallah JF, et al. Multiple genetic origins of histidine-rich protein 2 gene deletion in Plasmodium falciparum parasites from Peru. Sci Rep. 2013;3:2797.

27. Akinyi Okoth S, Abdallah JF, Ceron N, Adhin MR, Chandrabose J, Krishnalall K, et al. Variation in Plasmodium falciparum histidine-rich protein 2 (Pfhrp2) and Plasmodium falciparum histidine-rich protein 3 (Pfhrp3) gene deletions in Guyana and Suriname. PLoS One. 2015;10:e0126805.

28. Maltha J, Gamboa D, Bendezu J, Sanchez L, Cnops L, Gillet P, et al. Rapid diagnostic tests for malaria diagnosis in the Peruvian Amazon: impact of pfhrp2 gene deletions and cross-reactions. PLoS One. 2012;7:e43094.

29. Nkrumah B, Acquah SE, Ibrahim L, May J, Brattig N, Tannich E, et al. Comparative evaluation of two rapid field tests for malaria diagnosis: partec rapid malaria test $(R)$ and binax now $(R)$ malaria rapid diagnostic test. BMC Infect Dis. 2011;11:143. 